\title{
Scheduling and Flexible Control of Bandwidth and In-transit Services for End-to-End Application Workflows
}

\author{
Mehmet Fatih Aktas, Georgiana Haldeman and Manish Parashar \\ Rutgers Discovery Informatics Institute $\left(R D I^{2}\right)$ \\ Rutgers University, Piscataway NJ, USA Email: \{mehmet.aktas, haldeman, parashar\}@rutgers.edu
}

\begin{abstract}
End-to-end scientific application workflows that integrate high-end experiments and instruments with large scale simulations and end-user displays are becoming increasingly important. These workflows require complex couplings and data sharing between distributed components involving large data volumes and present varying hard (in-time data delivery) and soft (in-transit processing) quality of service (QoS) requirements. As a result, supporting efficient data transport is critical for such workflows. In this paper, we leverage software-defined networking (SDN) to address issues of data transport service control and resource provisioning to meet varying QoS requirements from multiple coupled workflows sharing the same service medium. Specifically, we present a flexible control and a disciplined resource scheduling approach for data transport services for science networks. Furthermore, we emulate an SDN testbed on top of the FutureGrid virtualized testbed and use it to evaluate our approach for a realistic scientific workflow. Our results show that SDN-based control and resource scheduling based on simple intuitive models can meet the requirements of the targeted workflows with high resource utilization.
\end{abstract}

Keywords: Advance scheduling, Data transport, SDN, In-transit processing, Distributed-area coupled simulation workflows

\section{Introduction}

As scientific discovery is becoming increasingly data driven, scientific applications are moving towards endto-end workflows that integrate coupled simulations with data sources such as instruments, sensor systems and experiments, and with data analysis and visualization pipelines to facilitate online knowledge extraction. Furthermore, the execution of such workflows often involves geographically distributed resources with runtime interactions, coordination and data exchanges between processes running on these resources $[1,2]$.

Scientific workflows typically involve complex couplings between the workflow components/services, requiring sharing of large data volumes with varying hard (in-time data delivery) and soft (in-transit processing) quality of service (QoS) requirements, and efficient data transport is a key requirement. Specifically, the time between when the data is generated at the producer and when it can be consumed at the consumer can have a significant impact on the execution of the workflow. For example, slower data delivery can throttle the consumer, or faster data delivery may require storing the data at the consumer. Some applications may require that data is delivered at the consumer within a tight time window (for example, data needed to control an experiment), which adds further requirements on the data transport. Additionally, there often exist natural mismatches in the way data is represented at producers and consumers, and data has to be transformed in a timely manner before it can be consumed. As a result, the data transport has to address multiple challenging requirements based on data sizes, data production and consumption rates, strict constraints on data delivery time and data storage, and managing data transformations between producers and consumers. The data transport medium is also typically shared by multiple application workflows with possibly competing application specific coupling requirements. Furthermore, best-effort solutions, which inherently can not offer service guarantees, will not be able to satisfy these requirements and achieve desired performance over utilized service medium [3].

The goal of this paper is to explore, prototype and evaluate a solution that can address these challenges, and enable the scheduling and control of bandwidth 
and in-transit services for end-to-end scientific application workflows. Specifically, in this paper, we leverage software-defined networking (SDN) to address issues of data transport service control and resource provisioning to meet varying QoS requirements from multiple coupled workflows sharing the same service medium. The specific contribution of this paper is a disciplined resource scheduling approach for data transport resources that is both application and network aware, and enables flexible control. The proposed control plane achieves flexibility by using SDN abstractions for managing the network, and further extending these abstractions to manage data transport services and schedule data transport resources. We also develop a model for in-transit data staging and data processing using intermediate resources in the data path using the approach outlined in $[2,4,5]$. Finally, we emulate an SDN testbed on top of the FutureGrid virtualized testbed and use it to evaluate our approach for an end-to-end Fusion workflow. Our results show that SDN-based control and resource scheduling based on simple intuitive models can meet the coupling requirement with high resource utilization.

The rest of the paper is organized as follows. Section 2 defines the problem and provides some background. Section 3 present our approach for scheduling and control of bandwidth and in-transit services for end-to-end scientific application workflows. Section 4 describes our emulation testbed and presents the experimental evaluation of our approach. Section 5 presents related work. Section 6 concludes the paper.

\section{Problem Description - Enabling End-to-End Coupled Simulation Workflows}

Emerging scientific applications integrate simulations with data sources such as experiments and instruments, and analysis and visualization pipelines, into end-to-end workflows. These workflows exhibit different and varying interaction, coordination and data coupling behaviors, such as:

- Tight Coupling: Coupled processes exchange data very frequently. Therefore, when tight coupling is dominant and processes are coupled over the network, total workflow execution time is typically dominated by the data transfer time. Often involves strict data transport constraints such as delivery time, data integrity and reliability.

- Loose Coupling: Relatively less frequent, asynchronous and possibly opportunistic data exchanges among the coupled processes. Producer and consumer progress at different rates, and may have different data representations making intermediate data transformation necessary.

- Dataflow Coupling: Data flows from producers to consumers using publish/subscribe/notify-like semantics, for example, in case of data processing and/or analysis pipelines.

It is essential for the data transport service to be application-aware and self-optimizing to meet requirements of such complex and varying coupling behaviors. Meanwhile, the overheads of service management on the application should be acceptable. In this paper, we focus on control and scheduling of data transfer (across network switches and links) nodes and in-transit service (intermediate staging and processing) hosts to meet varying data transport requirements of differing coupling behaviors, such as those described above. We propose a centralized advance scheduling system consisting of a centralized control layer and scheduler to enable workflow couplings. Enabling a coupling session requires scheduling a network path, and allocating resources on the scheduled path. Data transport resources that we consider are bandwidth and intermediate processing and staging capacity. Scheduler keeps the current state of the service medium, gets the coupling requirements from the applications, and generates scheduling and resource allocation rules upon receiving a scheduling request. These rules are then realized by the controller with a state setup process (Subsection 3.2).

A scheduling request consists of consumer address, size of data to be streamed and coupling requirements. Differing coupling behaviors translate into different requirements on the data transport service. For example, tight coupling usually has tight delivery time window while loose coupling takes place opportunistically or loose coupling requires more intermediate data manipulation than tight coupling. We formulated resource allocation as a disciplined optimization problem with the objective of maximizing cumulative satisfaction of coupling requirements. Scheduler numerically solves the formulated problem to optimize the resource allocation between coupling sessions (service users) (Subsection 3.3).

The Software Defined Networking (SDN) initiative aims at making network control simpler and more flexible using well-defined abstractions for forwarding and switch configuration. A key idea behind SDN is replacing the existing distributed control with centralized control. This is achieved by implementing network 
control programs on top of the network operating system (NOS). NOS provides the control programs with a global view of the network and interfaces for communicating with switches, enabling the control programs to configure the network state.

In this research, we explore how the data transport service control can benefit from SDN, and the capabilities that it provides, such as (1) open and programmable control, (2) faster innovation at the networking layer, (3) easy customization and optimization of network resource scheduling via flexible control. Overall, by leveraging SDN-based networking control, we propose an approach for the control and scheduling of data transport services shared by the end-to-end coupled application workflows.

\section{Scheduling and Flexible Control of Bandwidth and In-transit Services}

In this section, we explore scheduling and flexible control of bandwidth and in-transit services for end-toend scientific application workflows. We leverage SDN to address issues of data transport service control. We also present a disciplined formulation of resource provisioning to meet varying requirements from multiple coupled applications sharing the same service medium. Specifically, the solution we propose has three key components: (1) A flow-based in-transit service model, (2) Centralized layered architecture for data transport service control, and (3) Application and network aware resource scheduling for workflow couplings. In the following subsections, we will detail these three components.

\subsection{In-transit Service Model}

The goal of in-transit service is to offload (1) Opportunistic data transformation, (2) Data staging for justin-time data delivery, to available intermediate service hosts. This requires intervening the data flow between producers and consumers. Packet-based and flow-based approaches are two implementation of intermediate processing/staging.

End-to-end system design arguments draw the proper and modular boundaries between the service functions of distributed computer systems and communication networks [6]. End-to-end argument is a line of reasoning against low-level function (intermediate processing/staging in this case) implementation. This argument suggests that providing a function within the communication system itself should be avoided if the correct and complete implementation of the function depends on the application at the end points. Within the packetbased intermediate processing/staging model, network packets are intercepted on-the-fly for application specific goals, which violates the end-to-end system design arguments [7]. This violation of basic design principle of networks significantly impacts the existing network stack performance [8], [9].

We consider a flow-based intermediate processing/staging approach to avoid implementation of intransit service functions within network stack. In order to achieve flow-based in-transit service over the intermediate hosts, we propose a model that uses switchhost coordination, which is conceptually similar to the gateway-host coordination in the Phoebus architecture [10]. Brown et al. in [10] demonstrate that the Phoebus architecture preserves the end-to-end design arguments within sub-connections, which ensures that the overall data transport performance is not adversely affected. In the proposed flow-based approach, end-toend connections between producers and consumers are broken into sub-connections at intermediate hosts. Application data at these intermediate hosts is treated as a flow rather than packets, as illustrated in Figure 1. Autonomic switch-host coordination for creating and managing sub-connections is achieved by the granular forwarding control of SDN - this is further explained in Subsection 3.2.

Implementation of the proposed flow-based intermediate processing/staging consists of two main components: (1) In-transit service protocol (IT-SP) between the transport (TCP) and application layer, (2) In-transit processing/staging pipelines. IT-SP helps to identify data chunks to be processed/staged at the intermediate hosts. IT-SP header enables the flow of service specific information between the producer, the consumer and intermediate hosts. IT-SP header includes (a) Size of the IT-SP packet, (b) List of functions that are executed or to be executed over the data chunk. In-transit service pipelines execute scheduled processing/staging over the received data chunks. Switching between data flows service pipelines and processing rate and staging duration ${ }^{1}$ of service pipelines are managed according to intransit service tables. Entries of the service tables represent the state of the intermediate hosts, which is managed by the centralized controller as further discussed in Subsection 3.2.

In-transit service takes place over a data flow as follows. The producer encapsulates the application data

\footnotetext{
${ }^{1}$ Processing rate and staging duration of in-transit service pipelines are controlled using the conventional "Token Bucket Filter" (TBF) approach used for traffic control on packet-switching networks [11].
} 
stream with the IT-SP/TCP header. The intermediate hosts decapsulate the IT-SP/TCP header and execute programmed processing/staging pipelines on the data. When the in-transit pipelines finish executing, the resulting data is encapsulated using the updated ITSP/TCP header. Finally, the consumer decapsulates the IT-SP/TCP header and pushes the data to the application.

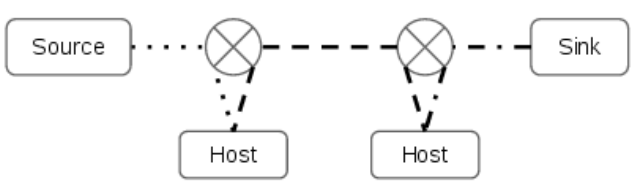

Figure 1: The end-to-end connection is broken into three subconnections, which are represented in the Figure by dotted, dashed and dotted-dashed lines.

\subsection{Data Transport Service Control}

We extend SDN-NOS to a Data Transport OS (DTOS) to offer support for in-transit service management. Control architecture consisting of the DT-OS and scheduler preserves the centralized and layered features of the SDN architecture. Similar to SDN-NOS, DT-OS provides a global view and centralized control of the service medium to the scheduler that uses this capability to optimize and achieve resource allocation (Figure 2). Control abstractions implemented by DT-OS enable autonomic switch-host coordination and efficient in-transit service management for flow-based intermediate processing and staging. Overall, DT-OS adds two additional abstractions to the existing SDN abstractions: (1) Switch-host coordination for flow-based intermediate data processing/staging and (2) Configuration of in-transit processing/staging pipelines at intermediate hosts.

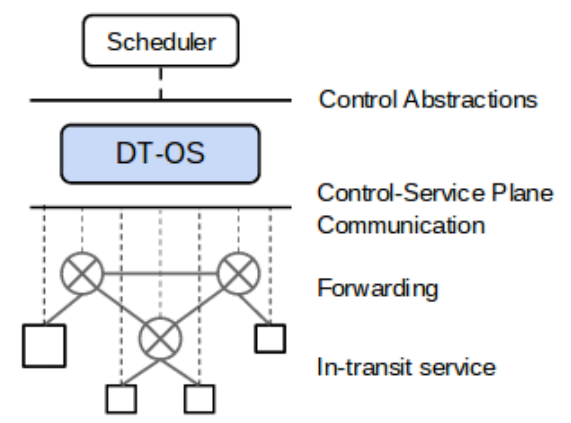

Figure 2: Data transport control architecture. Varying-sized squares depict heterogeneous processing/staging capacity of intermediate hosts.
We refer data flow between a producer-consumer application with certain application-level requirements as a coupling session. The flow of DT-OS operations for enabling a single coupling session is illustrated in Figure 3 , where the numbered steps are explained as follows:

- 1-Request: Application sends a request (UDP: sching_port), which consists of consumer address, size of data to be streamed and coupling requirements i.e., demand for in-time data delivery and intermediate processing. The gateway switch is programmed in advance to forward packets wildcarded with UDP: sching_port to the scheduler.

- 2-Scheduling: The scheduler does a feasibility check ${ }^{2}$ on the request and then generates rules for data walk route and resource allocation for the session. Data walk is the ordered list of network and in-transit service nodes that the data will walk through to reach consumer.

- 3-State setup: DT-OS translates the rules generated by the scheduler into low-level service state update messages, and sets up forwarding and intransit service tables at the switches and intermediate hosts.

- 4-Reply: The scheduler sends a reply (UDP: sching_port) over the gateway switch where the request was initially received, and the switch forwards it to source. The reply contains the scheduling information (e.g., scheduled TCP destination port stream_port, allocated bandwidth).

- 5-Data streaming: Once the source receives the reply, it immediately starts streaming data over ITSP/TCP: stream_port.

In step 3-State setup, DT-OS configures forwarding tables and in-transit service tables by communicating with switches and intermediate hosts over the control channels. DT-OS programs the switches to recognize session packets wild-carded with TCP: stream_port, modify destination and/or source IP/MAC addresses and forward them accordingly, in order to realize the scheduled data walk (over the source-host and host-sink sub-connections as shown in Figure 3). DT-OS also programs the in-transit hosts to listen on TCP:stream_port,

\footnotetext{
${ }^{2}$ If application request cannot be satisfied as is, they are modified appropriately and the application is notified e.g., transfer of given size of data cannot be finished within the requested time with available bandwidth.
} 
and execute scheduled processing/staging pipelines on the coupling data and forward it to the next hop.

The scheduler treats every application request as a new coupling session. When there are multiple active coupling sessions, the scheduler may need to reallocate resources between them, dynamically update the resource states, and inform the applications about any change.

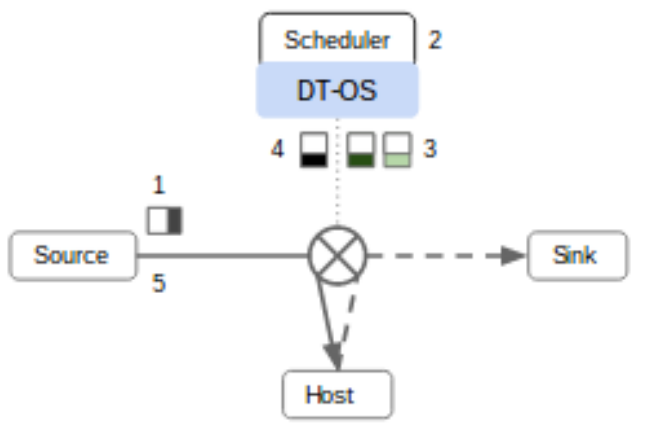

Figure 3: DT-OS operations for a single coupling session - an illustration of the control actions taken to schedule and implement a data walk for a coupling session between source and sink.

\subsection{Resource Allocation}

We have not yet addressed the problem of resource allocation which is crucial for efficient use of resources. In this part, we will show that opportunistic data transport service model allows us to reach a convex formulation of the resource allocation problem. Convex optimization problems have unique globally optimal solutions that can be obtained with several existing efficient, reliable and robust algorithms [12]. We implement the presented formulation as a control program in the scheduler which solves the resource allocation problem numerically for every scheduling run.

\subsubsection{General Overview}

Service is a generic term we use to describe capabilities provided by the network infrastructure, which are data transfer or in-transit data processing/staging. Capacity of these services is quantified with resources of data transfer rate, processing rate and staging duration. Network nodes providing these resources are network switches, links and in-transit hosts. Coupling sessions refer to executing coupled application data flow over the scheduled service nodes. Scheduling is the primary function of shared service management, and distributes finite available resources between coupling sessions.

The objective of scheduling is to maximize cumulative user satisfaction, which quantifies the satisfaction of user application requirements in terms of resource allocation distribution over the coupling sessions. To define it we use total penalty and utility functions $P, U$ : $R^{k \times N} \rightarrow R$ which return numerical penalty and utility scores for a particular resource allocation distribution. Furthermore, we define a penalty and utility function $P_{s}, U_{s}: R^{k} \rightarrow R$ for each coupling session $s$, which helps to formulate total penalty and utility functions as follows:

$$
\begin{aligned}
P(\mathbf{r}) & =\max _{s=1, \ldots, N} P_{s}\left(\mathbf{r}_{1, s}, \ldots, \mathbf{r}_{k, s}\right) \\
U(\mathbf{r}) & =\min _{s=1, \ldots, N} U_{s}\left(\mathbf{r}_{1, s}, \ldots, \mathbf{r}_{k, s}\right)
\end{aligned}
$$

where $\mathbf{r}_{i, s}$ denotes allocation of resource $i$ to coupling session $s$ and constitutes an element in the matrix $\mathbf{r} \in$ $R^{k \times N}$, where $k$ is the number of resources and $N$ is the number of coupling sessions. As promised in the last paragraph, we define scheduling objective of maximizing cumulative user satisfaction as minimizing the total penalty and maximizing total utility. Then, the problem of optimizing resource allocation can be expressed as:

$$
\begin{array}{cl}
\underset{w . r . t . R+}{\operatorname{minimize}} & f_{0}(\mathbf{r})=P(\mathbf{r})-\gamma U(\mathbf{r}) \\
\text { subject to: } & \operatorname{sum}(\mathbf{r}[i,:]) \leq C_{i}, i=1, \ldots, k, \\
& \mathbf{r} \geq 0 .
\end{array}
$$

where $C_{i}$ is total capacity of resource $i$ and $\gamma>0$ can be interpreted as the relative weight of total utility to total penalty.

In the next subsections, we define the individual components of the construction we just described and explain how they all fit together.

\subsubsection{Problem Domain-Specific Adaptation}

Fitting the problem of optimizing resource (bandwidth, processing rate and staging duration) allocation as formulated in the previous subsection ignores some important aspects. For example, resource allocation for coupling sessions assumes end-to-end connectivity, i.e., it assumes that there is a path from the producer to the consumer, and it abstracts all the aspects of routing involved in providing this end-to-end connectivity. Another requirement of resource allocation is data integrity. Guaranteeing data integrity requires some assumptions about the structure of the produced data. We assume that the produced data is in the form of list of chunks of uniform size. In addition, optimizing resource allocation over all the physical nodes (network switches, links and intermediate hosts) for all the coupling sessions - i.e. using $\mathbf{r}$ as the optimization variable matrix, is an overkill which would complicate the 
analytical formulation and cause much longer time for the solver to converge. As a result, for each coupling session, our scheduler schedules network links by selecting one of the available routing paths, and intermediate hosts on the chosen path, which together form the data walk. Then using convex optimization, optimal resource allocation over the scheduled data walk is obtained.

When there are multiple paths available to connect the session producer and consumer, our path selection approach attempts to choose the one that minimizes the overall discrepancy between the loads on all of the network links and in-transit hosts in the system. To explain it with an example, suppose a path should be assigned for a new coupling session and there are some number of available paths. Path selection algorithm picks a path and assumes the path resources are allocated for the session and finds the coefficient of variance (the ratio of standard deviation to the mean value) of fair bandwidth of all the network links and fair processing/staging capacity of all the in-transit hosts, and repeats this for every available path. Then, it finally picks the path which gives the minimum total coefficient of variance for overall fair bandwidth and fair processing/staging capacity. Fair capacity of a resource is calculated by dividing the total capacity with the number of coupling sessions using the resource.

However, simplifying the problem requires a mapping from the simplified problem of optimizing over the data walks to the more complex problem of optimizing over the physical nodes. Essentially, what we need is a mapping $\mathbf{M}$ of the previously defined matrix $\mathbf{r}$ (which abstracts the allocation over the physical nodes) to a matrix A (which abstracts the resource allocation over the data walks), i.e., we need to construct $\mathbf{A}$ and $\mathbf{M}$ to fit the equality: $\mathbf{A}=\mathbf{M r}$. We construct matrix $\mathbf{A}$ as:

$$
\mathbf{A}=\left[\begin{array}{cccc}
b w_{1} & b w_{2} & \ldots & b w_{N} \\
\operatorname{proc}_{1} & \operatorname{proc}_{2} & \ldots & \operatorname{proc}_{N} \\
d u r_{1} & d u r_{2} & \ldots & d u r_{N} \\
n_{1} & n_{2} & \ldots & n_{N}
\end{array}\right]
$$

where $b w_{s}, \operatorname{proc}_{s}, d u r_{s}$ represent allocation of bandwidth, processing rate and staging duration for session $s$, and $n_{s}$ is a variable to be assigned a value between 0 and 1 by the scheduler; the higher it is, the more processing work is scheduled to be completed in-transit for session $s$. In order to fit $\mathbf{A}=\mathbf{M r}$, we extend each element of $\mathbf{r}$ as a tuple $\left(r_{i, s}^{b w}, r_{i, s}^{p r o c}, r_{i, s}^{d u r}\right)$ such that, if $r_{i}$ is a network link then $r_{i, s}^{\text {proc }}, r_{i, s}^{d u r}$ are zero, and if it is an in-transit host then $r_{i, s}^{b w}$ is zero. So it follows that:

$$
\begin{aligned}
\text { bws } & =\min _{\forall i \mid r_{i} \in \text { link }} r_{i, s}^{b w} \\
\text { proc }_{s} & =\sum_{i \mid r_{i} \in \text { host }} r_{i, s}^{\text {proc }} \\
\text { dur }_{s} & =\sum_{i \mid r_{i} \in \text { host }_{s}} r_{i, s}^{\text {dur }}
\end{aligned}
$$

where link $k_{s}$ and host are sets of network links and intransit hosts on the data walk of session $s$.

We first set $\mathbf{M}$ as a constant per scheduling run (it is recalculated for each new scheduling run), set $\mathbf{A}$ as the optimization variable using which we can model the penalty and utility functions $P_{s}$ and $U_{s}$ in a straightforward manner, as presented in the next subsection. Based on this problem domain-specific modification, the formulation of the optimization problem defined in the previous subsection now becomes:

$$
\begin{array}{ll}
\operatorname{minimize} & P(\mathbf{A})-\gamma U(\mathbf{A}) \\
\text { subject to: } & \mathbf{A}=\mathbf{M r} \\
& \mathbf{A} \geq 0 \\
& {\left[n_{1} n_{2} \ldots n_{N}\right] \leq 1} \\
& \operatorname{sum}(\mathbf{r}[i,:]) \leq C_{i}, i=1, \ldots, k .
\end{array}
$$

When penalty function $P(\mathbf{A})$ is convex and utility function $U(\mathbf{A})$ is concave, problem formulated above becomes convex and it can be solved numerically using efficient solvers by the scheduler to optimize resource allocation.

\subsubsection{Penalty and Utility Functions}

Penalty and utility functions of a coupling session quantify the performance of scheduling in terms of its ability to meet the application requirements. They return penalty or utility scores given allocated resource and coupling requirements. Following the methodology suggested in [13], we define a requirement as hard if failing to satisfy it returns penalty, and as soft if succeeding to satisfy returns utility. In the workflows we have explored (1) there is a defined optimal time for data arrival, and a performance penalty is incurred if the data arrives earlier or later than the optimal time - ontime data delivery is a hard requirement, and (2) applications can benefit from on-the-fly data processing since it can lead to an earlier completion of workflow execution and/or reduced resource requirements, i.e., it incurs utility - in-transit processing is a soft requirement.

Overall, we model session penalty and utility as functions of allocated resource and application demands for hard and soft requirements. The required steps are as follows: 
Step 1 - Deriving models for session penalty and utility: To obtain a convex optimization objective (see equation (1) and (3)), $P_{s}$ and $U_{s}$ must be respectively convex and concave for each session.

Session penalty score with respect to the hard requirement (in-time delivery) can be modeled as the relative difference of the actual data transport time $\left(\right.$ trans $\left._{s}\right)$ from the optimal transport time (opttrans $\left.s_{s}\right)$ : $\mid 1-$ trans $_{s} /$ opttrans $_{s} \mid$ for session $s$. Transport completion time is the sum of data transfer, processing and staging times. Data transfer time increases multiplicatively with data size and additively with latency, and decreases multiplicatively with bandwidth, i.e., $D_{s} / b w_{s}+$ $L_{s}$ where $D_{s}$ is the size of streamed data and $L_{s}$ is the latency of the network path. The model for data transfer time is a convex function of $b w_{s}$ since bandwidth is always positive. Data processing time increases multiplicatively with data size, in-transit processing complexity, and decreases multiplicatively with processing rate, i.e., $D_{s} \times O_{s} \times\left(n_{s}\right)^{2} /$ proc $_{s}$ where $O_{s}$ is a scalar representing the total processing complexity calculated as the sum of complexity indexes of all processing tasks. Complexity index of a processing routine is a scale measuring how long it takes to execute a routine for unit data at unit processing rate and an oracle model for it can be obtained by running the task on data of varying sizes and interpolating the results. We chose to use $n_{s}^{2}$ as the percentage of processing tasks completed intransit ${ }^{3}$ to make the processing time model a quadraticover-linear function of $n_{s}$ and proc $_{s}$, i.e., the model is convex. Since running processing tasks that expand data on-the-fly increases the overall cost of data transport, we make the reasonable assumption that in-transit processing routines do not expand data. Therefore, the estimated data processing time by the presented model is an upper bound on the actual processing time. However, the gap between input and output data size of a processing task causes decrease in resource usage efficiency. Finally, total time that the streamed data is staged over in-transit nodes is represented by $d u r_{s}$. To summarize, we estimate the data transport completion time with the following model:

$$
\operatorname{trans}_{s}=\frac{D_{s}}{b w_{s}}+L_{s}+D_{s} \times \frac{O_{s} \times\left(n_{s}\right)^{2}}{\operatorname{proc}_{s}}+d u r_{s}
$$

However, substituting this model of trans $_{s}$ into session penalty model $\mid 1-$ trans $_{S} /$ opttrans $_{S} \mid$ cannot

\footnotetext{
${ }^{3}$ This does not disrupt anything since the value of optimization variable $n_{s}$ is constrained to be between 0 and 1 in equation (3), which constraints also $n_{s}^{2}$ in the same range i.e. if $n_{s}^{2}=0.4$, then $40 \%$ of the total processing work is scheduled to be completed in-transit.
}

achieve convexity because the absolute function is not non-decreasing. Therefore, we will introduce a new optimization variable $t_{s}$ for each session $s$ and replace trans $_{s}$ with it to get new convex session penalty model $\mid 1-t_{s} /$ opttran $_{s} \mid$, and add a new inequality to the optimization problem to reflect the relationship between trans $_{s}$ and $t_{s}$. Therefore, variable $t_{s}$ will trace epigraph of trans $_{s}$ :

$$
\frac{D_{s}}{b w_{s}}+L_{s}+D_{s} \times \frac{O_{s} \times\left(n_{s}\right)^{2}}{\text { proc }_{s}}+d u r_{s} \leq t_{s}
$$

The gap between $t_{s}$ and trans $_{s}$ is nonzero when available service capacity exceeds the capacity needed to achieve the optimal objective value. This is not an issue in our case since in data-intensive scientific workflows the network is typically over-utilized.

Session utility score with respect to the soft requirement (in-transit processing) could be estimated simply as the percentage of processing tasks completed intransit, which we modeled as $n_{s}^{2}$ previously. However, this would violate concavity of session utility function so instead we use square root of this; $n_{s}$ as the numerical session utility score.

Overall, both session penalty and utility models produce values between 0 and 1 and both roughly represent ratio of two numbers. Therefore, the objective function formulated in equation (3) makes sense since it returns the difference between two ratios.

Step 2 - Mapping application demands for hard and soft requirements on the models:. We will present a way to implement application demands for hard and soft requirements by setting a few well-understood parameters. To fit application demands on the models discussed in the previous step, we write session penalty and utility functions as follows:

$$
\begin{aligned}
P_{s} & =\max \left\{m_{s}^{\text {hard }} \times\left(\mid 1-t_{s} / \text { opttrans }_{s} \mid-\check{x}_{s}^{\text {hard }}\right), 0\right\} \\
U_{s} & =m_{s}^{\text {soft }} \times\left(n_{s}-\check{x}_{s}^{\text {soft }}\right)
\end{aligned}
$$

where:

- $m_{s}^{\text {hard }}$ : Rate of multiplicative increase in session penalty score per increase in the relative difference between the actual and optimal transport times.

- $\check{x}_{s}^{\text {hard: }}$ Tolerance for in-time delivery; session penalty becomes zero when relative difference between actual and optimal transport time is less than $\breve{x}_{s}^{\text {hard }}$.

- $m_{s}^{\text {soft }}$ : Rate of multiplicative increase in session utility score per increase in percentage of processing work done in-transit. 


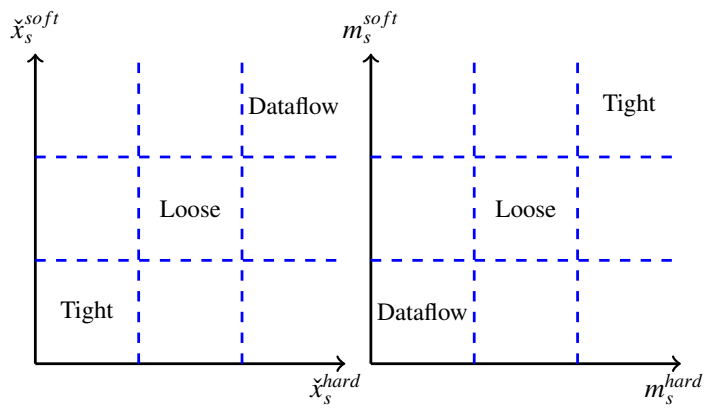

Figure 4: Mapping three different coupling types on two dimensional parameter space of $m_{s}^{\text {soft }}$ vs. $m_{s}^{\text {hard }}$ and $\breve{x}_{s}^{\text {soft }}$ vs. $\check{x}_{s}^{\text {hard }}$

- $\breve{x}_{s}^{\text {soft }}$ : Demand for in-transit processing; session utility becomes negative (penalty) when percentage of processing work done in-transit is less than $\breve{x}_{s}^{\text {soft }}$.

Overall, session penalty $P_{s}$ is convex and session utility $U_{s}$ is concave. The reason we used linear mapping function for session utility rather than piece-wise one as in session penalty, is to make $U_{s}$ concave. The only effect of this is that $\breve{x}_{s}^{\text {soft }}$ is a stronger parameter to increase demand for in-transit processing than $\breve{x}_{s}^{\text {hard }}$ is to decrease demand for in-time delivery - if desired, linear mapping function can also be used in penalty function.

Based on the coupling type, these four configuration parameters must be autonomously tuned for each session. Figure 4 illustrates the relationship between the coupling types outlined in section 2 and the four configuration parameters. In short, tight coupling has more strict time constraints, data flow has more opening for opportunistic in-transit processing and loose coupling is in the middle between these two. For practical implementation, the domain of the configuration parameters can easily be limited and discretized.

\section{Experiments and Results}

To evaluate the approach described in this paper, we used the "Plasma Disruption Analysis" workflow [14] from the $\mathrm{KSTAR}^{4}$ project to develop synthetic use case scenarios. In this application, plasma disruptions occur due to loss of stability and/or confinement of tokamak plasmas and cause a fast thermal and/or current quench within sub-milliseconds, which can damage the expensive (multi-billion dollars) tokamak device. As a result, finding precursors and early prediction of tokamak operation anomalies is a very active research field. One such

\footnotetext{
${ }^{4}$ Korea Superconducting Tokamak Advanced Research
}

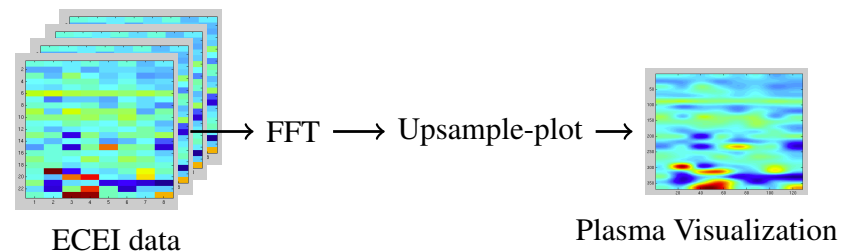

Figure 5: A schematic of the ECEI data and diagnostics workflow. The diagnostic routines do not change the size of the data, and comply with the optimization problem formulated in Subsection 3.3.

research effort is the plasma visualization diagnostics system designed to provide direct 2D/3D visualizations of the plasma in a tokamak. Visual plasma images are obtained via monitoring technologies such as Soft XRay (SXR), Microwave Imaging Reflectometry (MIR), or Electron Cyclotron Emission Imaging (ECEI). Diagnostic routines are then run over the plasma images to detect precursors of plasma disruption.

For our experiments, we used data, diagnostic and visualization routines for ECEI (see Figure 5) to compose synthetic use-case scenarios. ECEI generates high resolution 2D images of radiated electron temperature, which provide visualization of plasma instabilities specifically, a $24 \times 8$ float matrix image is generated every $2 \mu$ s, i.e., 5,000,000 images are generated in $10 \mathrm{sec}$ onds resulting in $\approx 3.5 \mathrm{~GB}$ of data. The over reaching goal of KSTAR is to enable a remote scientist, for example, in the US, to monitor the plasma visualizations to monitor tokamak stability and to take regulatory actions if necessary. This requires diagnostic and visualization routines to be run either at a US site or opportunistically over the available intermediate resources.

For our experimentation testbed, we implemented DT-OS by extending POX, which is a networking software platform [15], and the scheduler by using POX API. Furthermore, we used CVXPY [16] as the modeling language for the optimization problem for resource allocation. Numerical solution of the resource allocation is obtained using the solvers that CVXPY supports. Finally, we implemented the IT-SP layer on top of TCP at the producer, consumer and intermediate hosts.

We used mininet [17] to emulate a coupling session with producer-consumer pairs, network switches-links, and in-transit hosts. Running the entire system on a single machine is convenient but imposes limitation on switching and in-transit processing capacity. For example, if the machine has $3 \mathrm{GHz}$ of CPU, mininet can switch at most $3 \mathrm{Gbps}$ simulated traffic. Moreover, overall available emulation capacity is shared by multiple coupling sessions and resources. Therefore, we used 


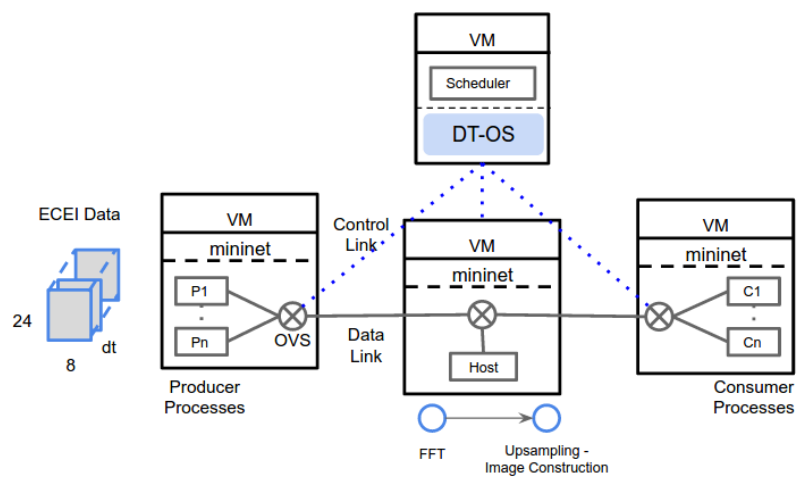

Figure 6: Schematic overview of the emulation testbed deployed across multiple VMs.

multiple mininets running on different VMs interconnected via GRE tunnels, as illustrated in Figure 6. We then deployed our testbed on a distributed OpenStack cloud as part of the FutureGrid testbed [18].

In our experiments, we used a use-case scenario which we obtained by monitoring the Fusion workflow, and adapted it appropriately for our virtualized emulation setup, which operates at a lower bandwidth (e.g. network links of $10 \mathrm{Mbps}$ ) and processing rate (e.g. intransit hosts of 100Mfps). We label the coupling sessions taking place during the workflow execution as tight, loose or dataflow based on the application characteristics i.e. demand for in-time delivery or in-transit processing. Relevant parameters of the scenario are summarized in Table 1, where arrival time is when application sends the scheduling request for the coupling session, data size is the size of data to be streamed over the coupling session and optimal transport time is the optimal time for data delivery.

\begin{tabular}{ccccc}
\hline $\begin{array}{c}\text { Session } \\
\text { Id }\end{array}$ & $\begin{array}{c}\text { Arrival } \\
\text { Time } \\
(\mathrm{sec})\end{array}$ & $\begin{array}{c}\text { Coupling } \\
\text { Type }\end{array}$ & $\begin{array}{c}\text { Data } \\
\text { Size } \\
(\mathrm{MB})\end{array}$ & $\begin{array}{c}\text { Optimal } \\
\text { Transport } \\
\text { Time }(\mathrm{sec})\end{array}$ \\
\hline 0 & 0 & Loose & 20 & 50 \\
1 & 10 & Dataflow & 15 & 60 \\
2 & 20 & Tight & 15 & 20 \\
3 & 30 & Dataflow & 60 & 80 \\
4 & 100 & Loose & 50 & 100 \\
5 & 110 & Dataflow & 50 & 100 \\
6 & 120 & Dataflow & 55 & 120 \\
7 & 130 & Loose & 80 & 150 \\
8 & 230 & Tight & 20 & 200 \\
9 & 240 & Dataflow & 20 & 200 \\
10 & 250 & Loose & 20 & 50 \\
\hline
\end{tabular}

Table 1: Parameters for the Fusion workflow scenario.

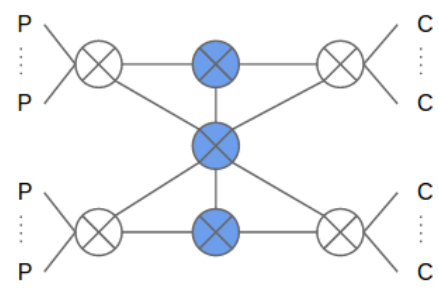

(a)

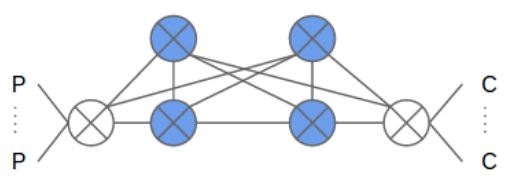

(b)

Figure 7: Two topologies used for the experiments. Filled switches are the ones with in-transit host connected.

We ran the experiments on two topologies shown in Figure 7. Performance results of the experiments are presented in Figure 8 and 9. Figure 8a and 9a show that as expected from the coupling models discussed in Section 3.3, the amount of processing completed in-transit is maximum for dataflow couplings and intermediate for loose and the least for tight coupling sessions. More specifically, session 1-2, 8-9 are dataflow-tight and 45 is loose-dataflow coupling pairs and the order for intransit processing completed is dataflow $>$ loose $>$ tight. Figure $8 \mathrm{~b}$ and $9 \mathrm{~b}$ show that the relative error between actual and optimal transport time can be slightly high for relatively short-duration couplings. This difference is mainly caused by the discrepancy between the estimated (by the models) and the actual values of data transport time. However the average error is approximately $5 \%$ which is acceptable for the Fusion workflow execution. Figure 8c and 9c show that scheduling overhead is also naturally higher for relatively short-duration couplings - in this case, inherent network link latency seems to have largest effect on the state setup time. Average overhead is close to $3 \%$ which does not disrupt Fusion workflow execution. Scheduling overhead can be completely masked by the application via sending scheduling request for a coupling session earlier than its actual starting time.

In the experiments, scheduling is initiated every time a new scheduling request is received. Each scheduling run uses all the resource capacities available to achieve its optimization goal. Figure 10 plots the logs of a network link and an in-transit host loads after every scheduling run; link capacity of $10 \mathrm{Mbps}$ and host capacity of $100 \mathrm{Mfps}$ are fully distributed between ac- 

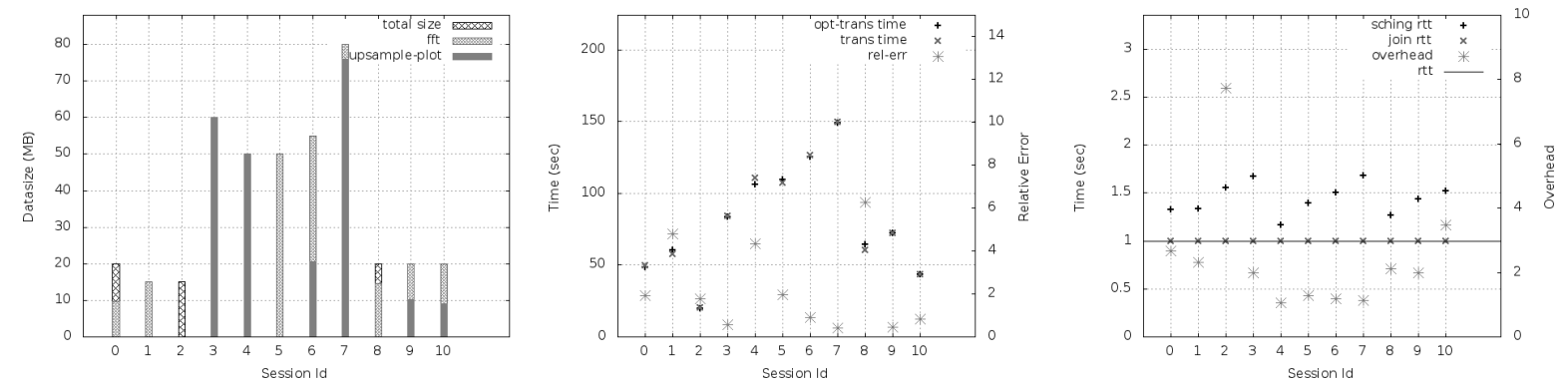

(a) Size of the total delivered data and the (b) Actual transport time: trans time, op- (c) Time between join request-reply: join size of the portions that are opportunisti- timal transport time: opt trans time and $r t t$ and scheduling request-reply: sching cally processed.

relative error between actual and optimal $r t t$. Scheduling overhead: overhead is the transport time: rel-err. relative error between sching rtt and actual data transport time. Horizontal line: $r t t$ is the round-trip time between session producer and scheduler due to network link latencies.

Figure 8: Results for the first set of experiments using the topology in Figure 7a.

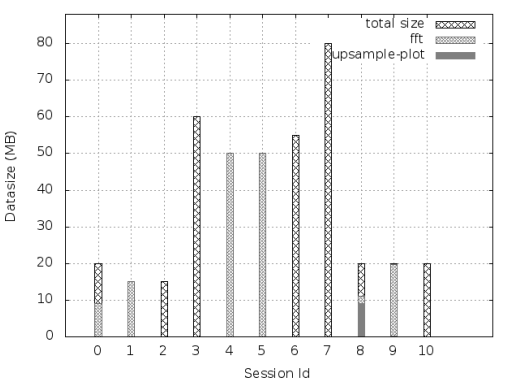

(a)

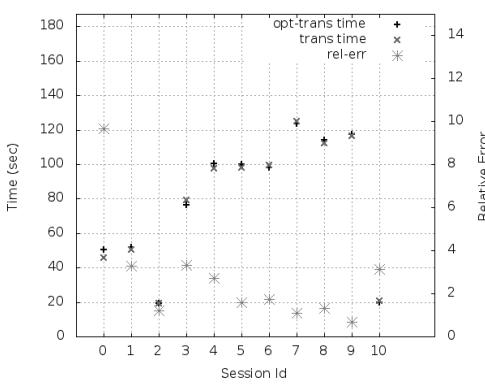

(b)

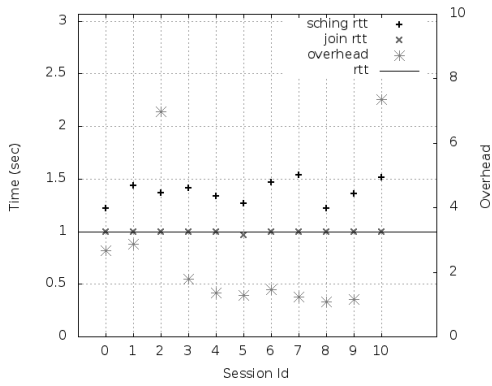

(c)

Figure 9: Results for the second set of experiments using the topology in Figure 7b.

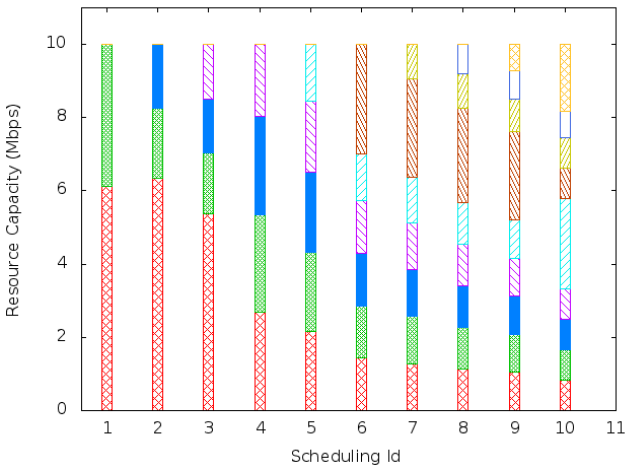

(a) Distribution of bandwidth allocation for a network link.

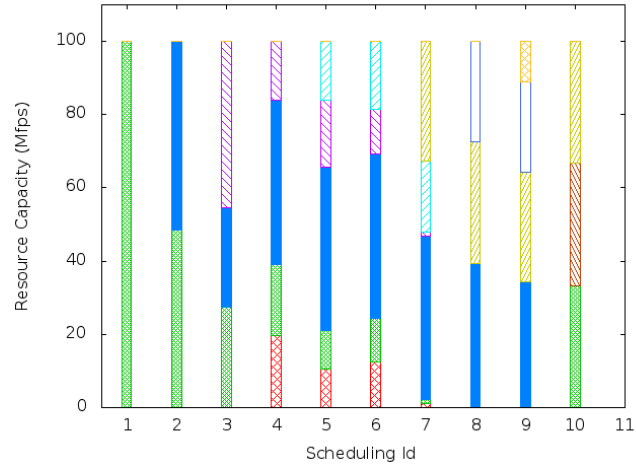

(b) Distribution of processing rate allocation for an in-transit host.

Figure 10: Distribution of the allocation of link and host capacities to active coupling sessions after each scheduling run. Every stacked bar corresponds to allocation to a single session. 
tive coupling sessions after every scheduling run. Note that since we assume the network is exclusively used by the scientific workflows and the bandwidth is sliced between different coupling sessions by the scheduler, even though resources are exhaustively used, there is no congestion in the network. This explains why the simple model to estimate transfer completion time used in Section 3.3 works very accurately - the TCP windows rapidly converge to the optimal (allocated bandwidth) without suffering from congestion.

To evaluate the introduced approach for a general use-case scenario, we run experiments using the topology in Figure $7 \mathrm{~b}$ with a random scenario. In the random scenario, coupling sessions arrive as a Poisson process of rate 0.04 ; one arrival every 25 seconds on average. Independent of the arrival process, each session is of one of the coupling types considered in this paper, i.e., tight, loose and dataflow. Also independent of the coupling type, every session streams data of size uniformly distributed between 10MB and 100MB, and has optimal transport time which is Gaussian given the data size. One realization of such scenario is given in Table 2 . Figure 11 shows the results. As in Fusion workflow scenarios, bandwidth, processing rate and staging capacity are distributed for each coupling session to maximize the specific demands in terms of in-time delivery and in-transit processing. Discrepancies between estimated and actual transport times are around $\% 5$ on average, scheduling overhead is around $\% 2$ on average.

\begin{tabular}{ccccc}
\hline $\begin{array}{c}\text { Session } \\
\text { Id }\end{array}$ & $\begin{array}{c}\text { Arrival } \\
\text { Time } \\
(\mathrm{sec})\end{array}$ & $\begin{array}{c}\text { Coupling } \\
\text { Type }\end{array}$ & $\begin{array}{c}\text { Data } \\
\text { Size } \\
(\mathrm{MB})\end{array}$ & $\begin{array}{c}\text { Optimal } \\
\text { Transport } \\
\text { Time }(\mathrm{sec})\end{array}$ \\
\hline 0 & 4 & Loose & 88 & 118 \\
1 & 40 & Dataflow & 71 & 106 \\
2 & 60 & Tight & 88 & 145 \\
3 & 80 & Dataflow & 67 & 95 \\
4 & 220 & Loose & 30 & 44 \\
5 & 238 & Dataflow & 70 & 108 \\
6 & 285 & Dataflow & 66 & 122 \\
7 & 293 & Loose & 84 & 125 \\
8 & 328 & Tight & 49 & 80 \\
9 & 335 & Dataflow & 62 & 101 \\
10 & 376 & Loose & 53 & 87 \\
\hline
\end{tabular}

Table 2: Parameters for the random scenario.

\section{Related Work}

In [5] Bhat et al. use adaptive buffer management at the end-points and in [2] use proactive and/or online user-defined policies for the QoS management of a self-managing data streaming and in-transit processing service for Grid-based data intensive workflows. In this work, the streaming and in-transit processing components work cooperatively (using feedforward and feedback messages) to meet overall application requirements and constraints. This solution is similar to our solution in that it uses QoS management to meet userdefined requirements. However, the approach in this work does not address the management of data transport resources, while our research is founded on scheduling and control of data transport resources. Cooperative management strategies presented in [2] could be used to extend our approach to work efficiently on a commodity service medium.

Active networking is a communication pattern [19] for tailoring network service to user requirements as explained in [20]. A network is called active when processing can be done within the network over active elements such as switches that have processing capability. Programming active switches according to application specific needs and taking advantage of packetbased processing within the network has been a key focus of active networking research. In [21], Lefevre et al. present an active network architecture (A-Grid) that attempts to provide QoS management for Grid data transport services in addition to other data transport services such as reliable multicast and dynamic service deployment. Their architecture employs QoS management at intermediate active routers, and in principal, it is similar to the opportunistic in-transit processing employed by our solution. However, our solution makes use of intermediate hosts to do flow-based processing rather than packet-based processing over active network elements. Moreover, their solution can only provide QoS management per application, but not per workflow, because their control architecture lacks a global view of the service medium and of simultaneously running user applications.

SDN as the centralized network management architecture has been actively explored for dynamic network resource management. A virtual single-switch abstraction on top of networks which employ both bandwidth reservation systems and SDN concepts is introduced in [22]. Using SDN control plane, a flow migration approach with dynamic resource scheduling and mapping of virtual networks to actual nodes is presented in [23]. Their formulation considers virtual network management cost as well as actual resource costs. Using the global network view and control provided by SDN, computation of end-to-end paths is formulated and implemented as a mixed integer linear programming problem in [24]. In [25] using SDN's centralized 


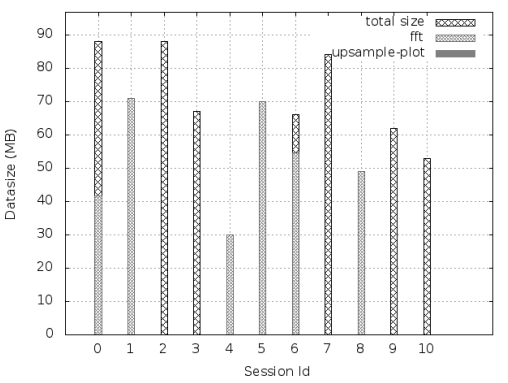

(a)

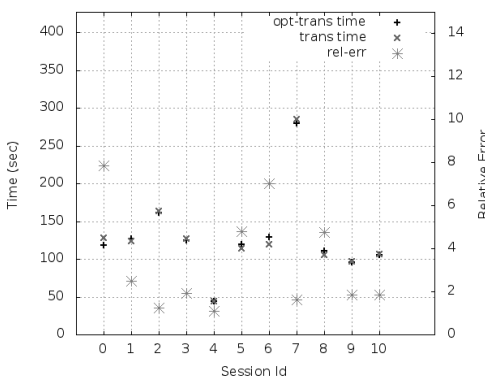

(b)

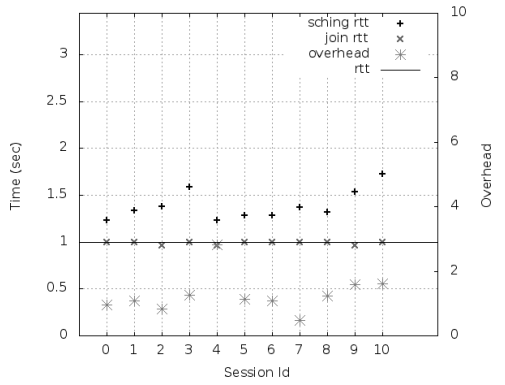

(c)

Figure 11: Results for the experiments using the random scenario in Table 2 and topology in Figure $7 \mathrm{~b}$.

control plane, authors present an approach to provide end-to-end QoS for video streaming applications using dynamic QoS routing. In order to manage different QoS requirements of applications sharing the same SDN network, a multi path routing (MPR) based approach is proposed in [26]. Basic principle of MPR is to divide application flows into sub-flows at the end-points where the number of the sub-flows together with the scheduled network paths determine the allocated network capacity to the application. Authors present MPR as a way of reaching a sub-optimal solution for resource allocation problem. Presented approach consists of two control plane components: (1) MPR agent (MPRA) at the end-points de/multiplexes between application flow and sub-flows, and detects QoS requirement violations, (2) SDN controller communicates with MPRA's and decides about allocation of new end-to-end paths to application flows by selectively picking the paths that are less occupied. Idea of deeply programmable network (DPN) is introduced in [27] as a network virtualization concept to implement programmability in not only control plane but also in data plane for customized implementation of data processing and communication protocols at the end-points and intermediate network nodes. In this paper, we implemented basic communication protocols between application end-points, intermediate hosts and control plane to realize in-transit service control and intermediate data processing/staging operations. Although this is an example of DPN, it is only a prototype implementation and aims to represent a use-case of SDN for enabling the end-to-end solution we presented for enabling workflow couplings.

Network resource reservation systems such as ESNET's OSCARS [28], UltraScience Net [29] and TeraPaths [30] provide on-demand channels for a certain time interval and guaranteed bandwidth. OSCARS establishes a virtual-circuit path for each user based on the service medium state and the reservation request received. If the requested bandwidth during the requested time interval cannot be provisioned by OSCARS' scheduler, user is informed only with a negative acknowledgment without any further suggestion. This makes users to send multiple consecutive requests as trial-and-error attempts to reach a request that can be granted and even so this would not be the optimal one that OSCARS can provide. In [31], Balman et al. present a flexible reservation scheme where users are returned possible optimal options e.g., earliest completion or shortest duration paths which are found using a novel path finding algorithm of quadratic complexity. The presented algorithm optimizes resource usage while meeting user constraints such as bandwidth and time interval for completion of data transfer.

Path finding algorithm in [31], and resource scheduling algorithms for QoS management presented in [32], [33], [34] schedule a network path with allocated bandwidth to guarantee user constraints and if they cannot be guaranteed the user is returned a failure message. Main difference of our approach from these advance bandwidth reservation systems is that we set the scheduling goal to optimize resource usage to meet application requirements opportunistically. This means giving up on guaranteed service performance but enables us to design a system that performs 'well-enough', which is sufficient from users' point of view in most cases [3]. Furthermore, problem of providing bandwidth guarantees in shared transport networks is NP-hard as shown in [32]. However, as presented in this paper since opportunistic resource allocation allows dynamic reallocation of the resources ${ }^{5}$ between users, it can be formulated as a convex optimization problem. Of course the price to

\footnotetext{
${ }^{5}$ Reallocation means changing allocated resource capacity on the scheduled path. Reallocation does not change scheduled path or service nodes.
} 
pay for dynamic reallocation is state setup time, which we tried to minimize using an SDN-based centralized control architecture.

\section{Conclusion}

This paper presented the architecture and design of a scheduling and control approach for data transport service management of data intensive scientific workflows. We leverage software-defined networking (SDN) to address issues of data transport service control and resource provisioning to meet varying QoS requirements from multiple coupled workflows sharing the same service medium. Furthermore, we addressed both, data transfer and in-transit processing/staging services. The presented approach has three key components: (1) A flow-based opportunistic in-transit processing/staging service model, (2) SDN-based centralized architecture for data transport service control, and (3) Application and service medium-aware scheduling. Overall, it attempts to address complex, dynamic and varying data transport requirements of coupled application workflows by scheduling bandwidth and in-transit processing/staging capacity. Scheduling is formulated by using intuitive models for data transfer and in-transit processing/staging, and implemented as a disciplined convex optimization problem.

The paper also presents an experimental evaluation using an emulated SDN testbed on top of the FutureGrid virtualized testbed; for the evaluation we used synthetic application workflow scenarios (derived from real $\mathrm{Fu}-$ sion simulations workflows and random processes) to demonstrate that presented approach can meet the coupling requirements and achieve high resource utilization.

\section{Acknowledgement}

The research presented in this work is supported in part by the US National Science Foundation (NSF) via grant numbers ACI 1339036, ACI 1310283, DMS 1228203, and IIP 0758566; by the Director, Office of Advanced Scientific Computing Research, Office of Science, of the US Department of Energy through the Scientific Discovery through Advanced Computing (SciDAC) Institute of Scalable Data Management, Analysis and Visualization (SDAV) under award number DE-SC0007455; by the Advanced Scientific Computing Research and Fusion Energy Sciences Partnership for Edge Physics Simulations (EPSI) under award number DE-FG02-06ER54857; by the ExaCT
Combustion Co-Design Center via subcontract number 4000110839 from UT Battelle; by the RSVP grant via subcontract number 4000126989 from UT Battelle; and by an IBM Faculty Award. The research was conducted as part of the NSF Cloud and Autonomic Computing (CAC) Center at Rutgers University and the Rutgers Discovery Informatics Institute (RDI2).

\section{References}

[1] L. Zhang, C. Docan, M. Parashar, The seine data coupling framework for parallel scientific applications, Advanced Computational Infrastructures for Parallel and Distributed Adaptive Applications (2010) 283.

[2] V. Bhat, M. Parashar, S. Klasky, Experiments with in-transit processing for data intensive grid workflows, in: Proceedings of the 8th IEEE/ACM International Conference on Grid Computing, IEEE Computer Society, 2007, pp. 193-200.

[3] M. Balman, S. Byna, Open problems in network-aware data management in exa-scale computing and terabit networking era, in: Proceedings of the first international workshop on Networkaware data management, ACM, 2011, pp. 73-78.

[4] V. Bhat, M. Parashar, H. Liu, M. Khandekar, N. Kandasamy, S. Abdelwahed, Enabling self-managing applications using model-based online control strategies, in: Autonomic Computing, 2006. ICAC'06. IEEE International Conference on, IEEE, 2006, pp. 15-24.

[5] V. Bhat, S. Klasky, S. Atchley, M. Beck, D. McCune, M. Parashar, High performance threaded data streaming for large scale simulations, in: Grid Computing, 2004. Proceedings. Fifth IEEE/ACM International Workshop on, IEEE, 2004, pp. 243-250.

[6] J. H. Saltzer, D. P. Reed, D. D. Clark, End-to-end arguments in system design, ACM Transactions on Computer Systems (TOCS) 2 (4) (1984) 277-288.

[7] S. Bhattacharjee, K. L. Calvert, E. W. Zegura, Active networking and the end-to-end argument, in: Network Protocols, 1997. Proceedings., 1997 International Conference on, IEEE, 1997, pp. 220-228.

[8] J. S. Plank, M. Beck, The logistical computing stack-a design for wide-area, scalable, uninterruptible computing, in: Dependable Systems and Networks, Workshop on Scalable, Uninterruptible Computing (DNS 2002), Citeseer, 2002.

[9] D. L. Tennenhouse, J. M. Smith, W. D. Sincoskie, D. J. Wetherall, G. J. Minden, A survey of active network research, Communications Magazine, IEEE 35 (1) (1997) 80-86.

[10] A. Brown, E. Kissel, M. Swany, G. Almes, Phoebus: A session protocol for dynamic and heterogeneous networks, University of Delaware, Tech. Rep 2008 (2008) 334.

[11] Token bucket filter, http://lartc.org/howto/lartc. qdisc.classless.html/.

[12] J. Mattingley, S. Boyd, Automatic code generation for real-time convex optimization, Convex optimization in signal processing and communications (2009) 1-41.

[13] S. L. Bird, B. J. Smith, Pacora: Performance aware convex optimization for resource allocation, in: Proceedings of the 3rd USENIX Workshop on Hot Topics in Parallelism (HotPar: Posters), 2011.

[14] J. Lee, J. Kim, C. Kessel, F. Poli, Simulation study of disruption characteristics in kstar, in: APS Meeting Abstracts, Vol. 1, 2012, p. 8047P.

[15] Pox, http://www.noxrepo.org/pox/about-pox/. 
[16] S. Diamond, E. Chu, S. Boyd, CVXPY: A Python-embedded modeling language for convex optimization, version 0.2 , http: //cvxpy.org/ (May 2014).

[17] B. Lantz, B. Heller, N. McKeown, A network in a laptop: rapid prototyping for software-defined networks, in: Proceedings of the 9th ACM SIGCOMM Workshop on Hot Topics in Networks, ACM, 2010, p. 19.

[18] G. Fox, G. von Laszewski, J. Diaz, K. Keahey, J. Fortes, R. Figueiredo, S. Smallen, W. Smith, A. Grimshaw, Futuregrida reconfigurable testbed for cloud, hpc and grid computing, Contemporary High Performance Computing: From Petascale toward Exascale, Computational Science. Chapman and Hall/CRC.

[19] D. L. Tennenhouse, D. J. Wetherall, Towards an active network architecture, in: DARPA Active NEtworks Conference and Exposition, 2002. Proceedings, IEEE, 2002, pp. 2-15.

[20] T. M. Chen, A. W. Jackson, Commentaries on" active networking and end-to-end arguments", Network, IEEE 12 (3) (1998) 66-71.

[21] L. Lefèvre, C.-d. Pham, P. Primet, B. Tourancheau, B. Gaidioz, J.-P. Gelas, M. Maimour, Active networking support for the grid, in: Active Networks, Springer, 2001, pp. 16-33.

[22] I. Monga, E. Pouyoul, C. Guok, Software defined networking for big-data science, SuperComputing 2012.

[23] R. Mijumbi, J. Serrat, J. Rubio-Loyola, N. Bouten, F. De Turck, S. Latré, Dynamic resource management in sdn-based virtualized networks, in: Network and Service Management (CNSM), 2014 10th International Conference on, IEEE, 2014, pp. 412417.

[24] R. Trivisonno, I. Vaishnavi, R. Guerzoni, Z. Despotovic, A. Hecker, S. Beker, D. Soldani, Virtual links mapping in future sdn-enabled networks, in: Future Networks and Services (SDN4FNS), 2013 IEEE SDN for, IEEE, 2013, pp. 1-5.

[25] H. E. Egilmez, S. T. Dane, K. T. Bagci, et al., Openqos: An openflow controller design for multimedia delivery with end-toend quality of service over software-defined networks, in: Signal \& Information Processing Association Annual Summit and Conference (APSIPA ASC), 2012 Asia-Pacific, IEEE, 2012, pp. $1-8$.

[26] E. Chemeritskiy, R. Smeliansky, On qos management in sdn by multipath routing, in: Science and Technology Conference (Modern Networking Technologies)(MoNeTeC), 2014 First International, IEEE, 2014, pp. 1-6.

[27] A. Nakao, Vnode: A deeply programmable network testbed through network virtualization, 3rd IEICE Technical Committee on Network Virtualization.

[28] C. Guok, D. Robertson, M. Thompson, J. Lee, B. Tierney, W. Johnston, Intra and interdomain circuit provisioning using the oscars reservation system, in: Broadband Communications, Networks and Systems, 2006. BROADNETS 2006. 3rd International Conference on, IEEE, 2006, pp. 1-8.

[29] N. S. Rao, W. R. Wing, S. M. Carter, Q. Wu, Ultrascience net: Network testbed for large-scale science applications, Communications Magazine, IEEE 43 (11) (2005) S12-S17.

[30] B. Gibbard, D. Katramatos, D. Yu, Terapaths: end-to-end network path qos configuration using cross-domain reservation negotiation, in: Broadband Communications, Networks and Systems, 2006. BROADNETS 2006. 3rd International Conference on, IEEE, 2006, pp. 1-9.

[31] M. Balman, E. Chaniotakis, A. Shoshani, A. Sim, A flexible reservation algorithm for advance network provisioning, in High Performance Computing, Networking, Storage and Analysis (SC), 2010 International Conference for, IEEE, 2010, pp $1-11$.

[32] S. Sharma, D. Katramatos, D. Yu, End-to-end network qos via scheduling of flexible resource reservation requests, in: Proceedings of 2011 International Conference for High Performance Computing, Networking, Storage and Analysis, ACM, 2011, p. 68.

[33] L. Shi, S. Sharma, D. Katramatos, D. Yu, Scheduling end-toend flexible resource reservation requests for multiple end sites, in: Computing, Networking and Communications (ICNC), 2015 International Conference on, IEEE, 2015, pp. 810-816.

[34] T. Orawiwattanakul, H. Otsuki, E. Kawai, S. Shimojo, Dynamic time scheduling in advance bandwidth reservation, in: Advanced Information Networking and Applications Workshops (WAINA), 2012 26th International Conference on, IEEE, 2012, pp. $885-890$. 